\title{
A Dual-Aggressive Model of Tumor-Immune System Interactions
}

\author{
https://doi.org/10.3991/ijoe.v15i10.10877 \\ Abdulkareem Ibrahim Afolabi $\left.{ }^{(}\right)$, Normah Maan \\ Universiti Tecknologi Malaysia \\ systemania12@gmail.com
}

\begin{abstract}
Biomedical literature suggested that the tumor-immune system physical phenomenon usually climaxes into either tumor elimination or escape. In retort to the phenomenological mechanics of tumor-immune system interaction, researchers had used Mathematical models mostly prey-predator and competitive extensively, to model the dynamics of tumor immune system interaction. However, these models had not accounted for total elimination and, or escape of tumor as hypothesizes by immunoediting hypotheses. In this work, we propose a dual aggressive model based on the biological narration of tumorimmune system interactions. The stability analyses of tumor-negative steady state are stable if the rate at which body cells dies is less than their proliferation rate a confirmation of biological listed causes of the tumor. The tumor-positive steady state is always unstable and saddle with the likelihood of either elimination or escape of tumor. Numerical analysis validates our analytical results and provides insight into the dynamics of the benignant and malignant tumor. The immunosuppression by tumor is not only visible but also validated by both analytical and numerical analysis.
\end{abstract}

Keywords-Tumor, Immune System, Immunoediting, Immunosurveillance, Prey-Predator Model and Competitive Model

\section{$1 \quad$ Introduction}

Tumor-immune system interaction had revolutionized, From the era of immunosurveillance that hypothesizes immune system capacity to recognize and eliminate nascent malignant cells [1], to the era of immunoediting which hypothesizes that the interaction might be responsible for both elimination and sculpting of the immunogenic phenotype of tumor that eventually formed in the immunogenic host [2\{Vesely, 2011 \#7. The immunoediting hypothesized possible outcomes of the interaction include elimination phase a state of pathogens' elimination. The equilibrium phase is a state where anti-tumor immunity control tumor growth but does not eliminate it and tumor cell appears dormant $\{$ Mittal, $2014 \# 8,3]$. The last phase is the escape phase, a state where the immunosuppressive mechanism allows tumor cell to escape from control and grow in an unrestricted manner [4-6]. "The interactions between the im- 
mune system and tumor happen through complex events that usually eventually climax either in successful tumor eradication or immune evasion" [7]

The critical demands in immunology and oncology are knowing how the immune system affects cancer. These demands had prompted many mathematical models research work on tumor-immune system interaction using either prey-predator or competitive model as in [1, 8-12] and others who had built on these works. Most of these authors had used interchangeably different growth model for tumor growth. However, the choice of model for tumor growth had always been discretionary as the choice ranges from the simple to a complex with linear or exponential form. Most complex models selected by the modelers contain either exponential and linear phases or takes into account the process of growth or the sigmoidal models with the assumption that the carrying capacity is dynamic (time-dependent). Another selection is with the programmed cell death to factor the tumor loss volume as discussed in [13].. The need for a new mathematical model with precision was recommended in $[14,15]$. Recently, a differential equation model was proposed for tumor growth in $[16,17] \mathrm{e}\}$ which demonstrate the universality of known tumor growth models properties.

Here, we briefly reviewed some of the mostly referenced models of tumor-effector cells dynamics both the prey-predator and competition model version.. [10] used a competitive model to describe the binding and detaching of effector cell and tumor cells without damaging cells. The qualitative behaviors of their model, using bifurcation theory accounted for tumor dormancy and sneak through.. Kuznestov et al. submitted that, their work do not account for total elimination even with highly immungenic tumors. Panettal et al. in [11] proposed a three population model describing the interaction effector cells, tumor cells and cytokrn IL-2. They used logistic model for tumor growth. The stability analysis of their model accounted for an always unstable and saddle trivial equilibrium ie when all the populations are zeros and three nontrivial steady states. Using bifurcation diagram their findings revealed that with low antigen there is possibility of large mass of tumor or dormancy of tumor[1] provided extension to[11] with inclusion of process of activation and maturation of effector cells to describe the competition for resources between the tumor and effector cells. Their analysis without delay predicted tumor dormancy or victory. Sotolongo et al in [18] improved the model proposed by Bell in [19] with the inclusion of "the death of lymphocytes due to the increase of malignant cells population, the flux of lymphocytes towards the place of local interaction and the effect produced by the application of cytokine doses". Their work displays brief tumor oscillations and elongated tumor relapse. Gallach [9] modified the work proposed in [10] by replacing the MichaelisMenten form in [10] with a Lotka-Volterra form, a prey-predator like model. Their analysis without delay accounted for tumor dormancy only.

Eftimie et al in [16] reviewed extensively ordinary differential equations model of tumor-immune system interaction which included the interaction of tumor and families of immune cells, cell mediated tumor growth and immunotherapies in form of two, three, four and five equations interaction Models. They affirmed that these entire models do exhibit tumor dormancy and oscillation. It is evident in all the works cited above that both prey-predator and competitive models had not accounted for tumorelimination or escape. In this work, we proposed a dual aggressive model based on 
biomedical narration to model tumor-immune system physical phenomenon and the stability analyses of the steady states were obtained. Numerical simulation was used to validate our analytical results using relevant and recent biological parameter values.

\section{Model Formulation}

Biomedical studies have it that, the outcome of tumor-immune interactions is supported by a number of highly unrelated parameters such as tumor "foreignness" (immunosurveillance) and T-cells inhibitory mechanisms (immunosuppression) [20]. We begin the formulation of our model by considering the immune system interaction with tumor cells with tendencies of recognition and elimination of tumor cells and sculpt of the cell phenotype leading to a less immunogenic variant that facilitates tumor growth and immune evasion. The tumor-immune system interaction is described by equation (1), the inclusion tumor immunosurpressive mechanism is an unusual feature in our model, making the model dual aggressive.

$$
\begin{gathered}
\frac{d E}{d t}=\alpha E(t)+\beta E(t) T(t)-\theta E(t) \\
\frac{d T}{d t}=\delta T(t)+\gamma E(t) T(t)-\vartheta T(t)
\end{gathered}
$$

Where the first equation of (1) represent the rate of changes in population density of effector cells over time which is the sum of the proliferation rate of effector cells $\alpha$ and the immunosurvellance rate $\beta$ to eliminate tumor cells minus the deactivate or death rate $\theta$ of effector cells. The second equation of (1) is the rate of changes in population density of tumor cells over time which is the sum of proliferation rate $\delta$ of the tumor and the inhibitory rate $\gamma$ of effector cells by the tumor minus the death rate $\vartheta$ of the tumor cells.

The population of tumor in equation1 is modeled by a growth model defined as singled out in equation 2 to effectively remove the problem of choice arising from collection of growth models like logistic, gompertz, power models and others. This growth model harmonized other tumor growth models as acknowledged in \{d’Onofrio, $2005 \# 30\}$ \{Eftimie, 2011 \#26\}\{Wilkie, $2013 \# 31$ \}.

$$
\frac{d T}{d t}=\delta T(t)-\vartheta T(t)
$$

Equation 2 gives a good basis to determine the tendencies of tumor growth as $\delta T(t)>\vartheta T(t)$ indicates growth of tumor over time, $\delta T(t)<\vartheta T(t)$ indicates decay of tumor over time and $\delta T(t)=\vartheta T(t)$ indicates tumor dormancy.

To describe the points at which the interaction brought about an unchanged population for the tumor and effector cells, when seek to determine the steady states where the populations remain unchanged. The behaviors of the model near these steady states are interest in determining the stability status of the model. We considered the steady states and their stability in the next section. 


\section{$3 \quad$ Steady States and Stability Analysis}

The equation 1 has two biological relevant steady states namely, tumor-negative $\left(E^{*}, T^{*}\right)=(0,0)$ where both population are zeros and tumor-positive $\left(E^{*}, T^{*}\right)$ $=\left(\frac{(\vartheta-\delta)}{\gamma}, \frac{(\theta-\alpha)}{\beta}\right)$ where both population are present. By linearizing equation 1 , the characteristic equation has the form

$$
\lambda^{2}+\left(\left[\vartheta-\delta-\gamma E^{*}\right]+\left[\theta-\alpha-\beta T^{*}\right]\right) \lambda+\left(\delta+\gamma E^{*}-\vartheta\right)\left(\alpha+\beta T^{*}-\theta\right)-
$$

$\beta E^{*} \gamma T^{*}=0$

Lemma: The tumor negative steady state $\left(\mathrm{E}^{*}, \mathrm{~T}^{*}\right)=(0,0)$ is stable, if $\alpha<\theta$ and $\delta<\vartheta$.

Proof: Substituting $\left(\mathrm{E}^{*}, \mathrm{~T}^{*}\right)=(0,0)$ into (3) we have

$$
\lambda^{2}+([\vartheta-\delta]+[\theta-\alpha]) \lambda+(\delta-\vartheta)(\alpha-\theta)=0
$$

Thus,

$$
\lambda_{1}=-(\vartheta-\delta), \quad \lambda_{2}=-(\theta-\alpha)
$$

The tumor-negative steady state $\left(\mathrm{E}^{\wedge *}, \mathrm{~T}^{\wedge *}\right)=(0,0)$ is stable if the proliferation rate of body cells is less than or equal to their death rate, otherwise it is unstable.

Lemma: The tumor positive steady state $\left(\mathrm{E}^{*}, \mathrm{~T}^{\wedge *}\right)=\left(\frac{(\vartheta-\delta)}{\gamma}, \frac{(\theta-\alpha)}{\beta}\right)$ is always unstable and saddle.

Proof: Substituting $\left(\mathrm{E}^{*}, \mathrm{~T}^{*}\right)=\left(\frac{(\vartheta-\delta)}{\gamma}, \frac{(\theta-\alpha)}{\beta}\right)$ into (3) we have,

$$
\begin{aligned}
& \lambda^{2}-(\theta-\alpha)(\vartheta-\delta)=0 \\
& \lambda^{2}= \pm \sqrt{(\theta-\alpha)(\vartheta-\delta)}
\end{aligned}
$$

The tumor-positive steady states $\left(\mathrm{E}^{*}, \mathrm{~T}^{\wedge *}\right)=\left(\frac{(\vartheta-\delta)}{\gamma}, \frac{(\theta-\alpha)}{\beta}\right)$ is unstable saddled. The interaction yields a dynamical situation which might climax into either tumor elimination or escape. $\|$

To see long time behavior of the model we employed the Dulac Bendixson criteria as in \{Gasull, $2013 \# 32\}\{$ Ibáñez, $2013 \# 33\}$ to determine the periodic status of the solution of (1) we use the auxiliary function $\frac{1}{E(t) T(t)}$ and the equation (1) takes the form

$$
\frac{\partial\left(\frac{\alpha E(t)+\beta E(t) T(t)-\theta E(t)}{E(t) T(t)}\right)}{\partial E}+\frac{\partial\left(\frac{\delta T(t)+\gamma E(t) T(t)-\vartheta T(t)}{E(t) T(T)}\right)}{\partial T}=0
$$

Going by (6), it translates that the model (1) does not have periodic. 


\section{$4 \quad$ Numerical Simulation}

In this section, we aim to validate our analytical results numerically. we selected from the recent work, biological meaningful parameter values as indicated in Table 1.

Table 1. Model Biological Parameters Values

\begin{tabular}{|c|c|l|l|}
\hline Parameters & \multicolumn{1}{|c|}{ Values } & \multicolumn{1}{|c|}{ Units } & \multicolumn{1}{c|}{ References } \\
\hline$\alpha$ & 0.1811 or 16 & day $^{-1}$ & $\{$ Kuznetsov, 1994 \#16\}\{Mempel, 2004 \#\} \\
\hline$\beta$ & 2.9 & $n g m l^{-1}$ & $\{$ Robertson-Tessi, 2012 \#35\}\{Thomas, 2005 \#36\} \\
\hline$\theta$ & 1 & $d a y^{-1}$ & $\{$ Yates, 2001 \#37\} \\
\hline$\delta$ & $0.1-1$ & $d a y^{-1}$ & $\{$ Mempel, 2004 \#34\} \\
\hline$\gamma$ & 3,5 & $n g m l^{-1}$ & $\{$ Robertson-Tessi, 2012 \#35\} \\
\hline$\vartheta$ & 0.9 & $d a y^{-1}$ & $\{$ Dudley, 2002 \#38\} \\
\hline
\end{tabular}

Results obtained in previous sections are governed by the relation of death and proliferation rates of tumor and effector cells. This is mathematically expressed as $(\theta>$ $\alpha)$ and $(\vartheta>\delta)$ for growth of effector cells and tumor cells respectively and $(\theta<$ $\alpha)$ and $(\vartheta<\delta)$ ) for their decays. We simulated our models in these two scenarios to obtain a qualitative behavior of the model using parameters in Table 1.

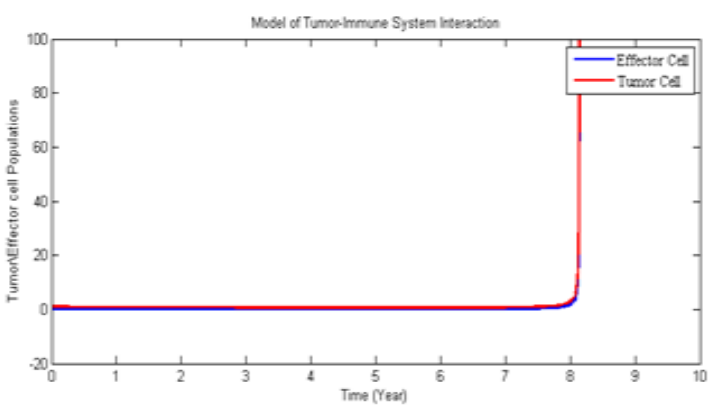

Fig. 1. Quantative solution of Equation

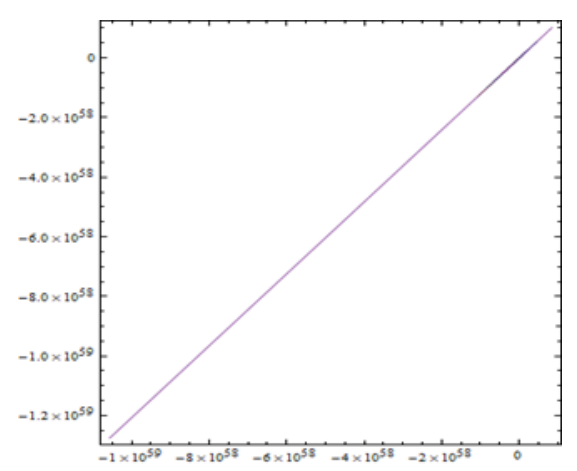

Fig. 2. Phase portrait for equation 
Fig. 1 is the quantitative solution of equation (1) when the death rates of tumor and effector cells are less than their respective growth rate with the following model parameter values $\alpha=0.1811, \beta=2.9, \theta=1, \delta=0.8, \gamma=3.5$ and 0.9 . A stiff combative interaction that keeps tumor dormant was observed for almost nine months since the emergent of tumor until the effector cells showed weakness and tumor escape uncontrollably. In Fig. 2, we obtained phase portrait for equation (1) under the same situation as in Fig, and the simulation revealed the existence of a whole stable region. A confirmation of the analytical result obtained in equation (5).

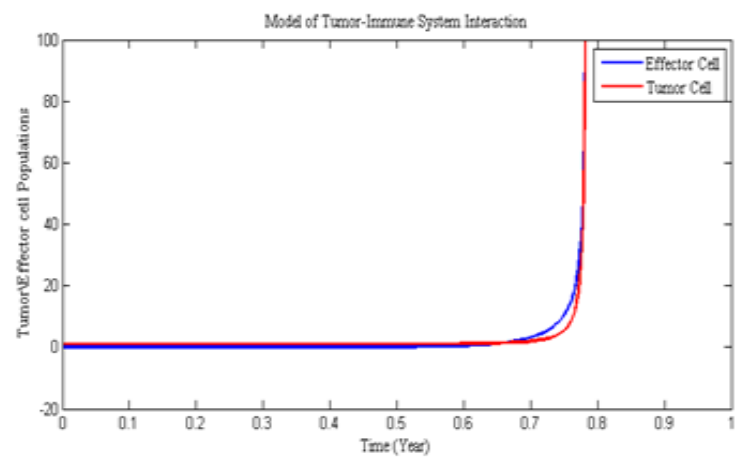

Fig. 3. quantitative solution of equation

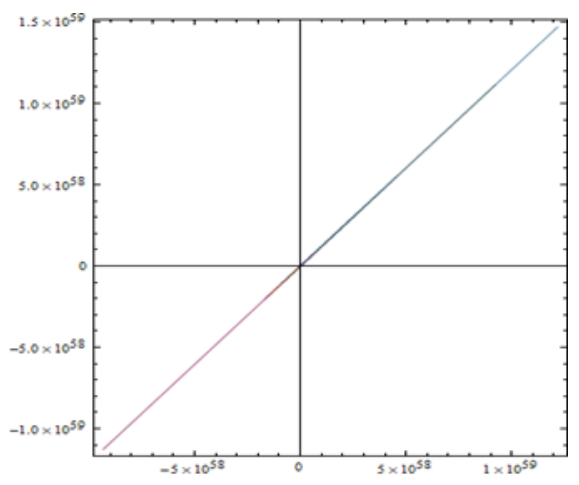

Fig. 4. phase portrait for equation

Fig. 3, is a quantitative solution equation (1) when the proliferation rate of both the tumor and the effector cells are greater than their death rate with the following model parameter values $\alpha=16, \beta=2.9, \theta=1, \delta=1, \gamma=3.5$ and 0.9. It exhibits a longer combative interaction comparing to Fig, 1 resulting to tumor escape. Though an intermittent reappearance of effector cells is later recorded it doesn't stop the escape of tumor cells. Fig, 4 is a phase portrait for equation (1) under the same parameter values and the simulation revealed the existence of small stable region and a larger unstable region. These confirmed the unstable status of equation (5). 


\section{Discussion}

In this paper, we had proposed a dual aggressive model of tumor-immune system interaction bearing in mind the immunoediting hypotheses. The tumor-negative steady state is stable when the body cells do not proliferate excessively and the tumorpositive steady is always unstable and might either climaxes into either tumor elimination or escape. We used phase portrait to reflect a possible outcome of the interaction in two scenarios:

1. When the proliferation rate of tumor and effector cells are less than their death rates which yields the decay of tumor (stable situation)

2. When the proliferation rate of tumor and effector cells are greater than their death rates which suggested a partial decay and growth of tumor (unstable situation) see Fig. $1 \& 2$.

The numerical simulation also exhibited the immunosuppression with the intermittent reappearances of effector cells. In our next work, we will consider the introduction of time delay to model the natural processes of the interaction.

\section{Acknowledgement}

The authors would like to acknowledge the Ministry of Education Malaysia and TRGS grant with Vol. no. 4L854. And equally, Federal Polytechnic Kaura-Namoda, Nigeria for their support.

\section{$7 \quad$ References}

[1] Burić N, Todorović D. Dynamics of delay-differential equations modelling immunology of tumor growth. Chaos, Solitons \& Fractals. 2002;13(4):645-55. https://doi.org/10.1016/s0960-0779(00)00275-7

[2] Dunn GP, Old LJ, Schreiber RD. The three Es of cancer immunoediting. Annu Rev Immunol. 2004;22:329-60. https://doi.org/10.1146/annurev.immunol.22.012703.104803

[3] Almog N. Molecular mechanisms underlying tumor dormancy. Cancer letters. 2010;294(2):139-46. https://doi.org/10.1016/j.canlet.2010.03.004

[4] Manjili MH. Revisiting cancer immunoediting by understanding cancer immune complexity. The Journal of pathology. 2011;224(1):5-9. https://doi.org/10.1002/path.2865

[5] Reiman JM, Kmieciak M, Manjili MH, Knutson KL, editors. Tumor immunoediting and immunosculpting pathways to cancer progression. Seminars in cancer biology; 2007: Elsevier. https://doi.org/10.1016/j.semcancer.2007.06.009

[6] Dunn GP, Fecci PE, Curry WT. Cancer immunoediting in malignant glioma. Neurosurgery. 2012;71(2):201-23. https://doi.org/10.1227/neu.0b013e31824f840d

[7] Arum C-J, Anderssen E, Viset T, Kodama Y, Lundgren S, Chen D, et al. Cancer immunoediting from immunosurveillance to tumor escape in microvillus-formed niche: a study of syngeneic orthotopic rat bladder cancer model in comparison with human bladder cancer. Neoplasia. 2010;12(6):434-42. https://doi.org/10.1593/neo.91824 
[8] Foryś U. Marchuk's model of immune system dynamics with application to tumour growth. Computational and Mathematical Methods in Medicine. 2002;4(1):85-93.

[9] Gałach M. Dynamics of the Tumor---Immune System Competition---the Effect of Time Delay. International Journal of Applied Mathematics and Computer Science. 2003;13:395406.

[10] Kuznetsov VA, Makalkin IA, Taylor MA, Perelson AS. Nonlinear dynamics of immunogenic tumors: parameter estimation and global bifurcation analysis. Bulletin of mathematical biology. 1994;56(2):295-321. https://doi.org/10.1016/s0092-8240(05)80260$\underline{5}$

[11] Kirschner D, Panetta JC. Modeling immunotherapy of the tumor-immune interaction. Journal of mathematical biology. 1998;37(3):235-52.

[12] d'Onofrio A. A general framework for modeling tumor-immune system competition and immunotherapy: Mathematical analysis and biomedical inferences. Physica D: Nonlinear Phenomena. 2005;208(3-4):220-35. https://doi.org/10.1016/j.physd.2005.06.032

[13] Evain S, Benzekry S. Mathematical modeling of tumor and metastatic growth when treated with sunitinib: Inria Bordeaux Sud-Ouest; 2015.

[14] Sápi J, Drexler DA, Kovács L, editors. Comparison of mathematical tumor growth models. 2015 IEEE 13th International Symposium on Intelligent Systems and Informatics (SISY); 2015: IEEE. https://doi.org/10.1109/sisy.2015.7325403

[15] Murphy H, Jaafari H, Dobrovolny HM. Differences in predictions of ODE models of tumor growth: a cautionary example. BMC cancer. 2016;16(1):163. https://doi.org/10.1186/s12885-016-2164-x

[16] Eftimie R, Bramson JL, Earn DJ. Interactions between the immune system and cancer: a brief review of non-spatial mathematical models. Bulletin of mathematical biology. 2011;73(1):2-32. https://doi.org/10.1007/s11538-010-9526-3

[17] Enderling H, Chaplain MA, Hahnfeldt P. Quantitative modeling of tumor dynamics and radiotherapy. Acta biotheoretica. 2010;58(4):341-53. https://doi.org/10.1007/s10441-0109111-Z

[18] Sotolongo-Costa O, Molina LM, Perez DR, Antoranz J, Reyes MC. Behavior of tumors under nonstationary therapy. Physica D: Nonlinear Phenomena. 2003;178(3-4):242-53. https://doi.org/10.1016/s0167-2789(03)00005-8

[19] Bell GI. Predator-prey equations simulating an immune response. Mathematical Biosciences. 1973;16(3-4):291-314. https://doi.org/10.1016/0025-5564(73)90036-9

[20] Foppen MHG, Rozeman EA, van Wilpe S, Postma C, Snaebjornsson P, van Thienen JV, et al. Immune checkpoint inhibition-related colitis: symptoms, endoscopic features, histology and response to management. ESMO open. 2018;3(1):e000278.

https://doi.org/10.1136/esmoopen-2017-000278

\section{Authors}

Abdulkareem Ibrahim Afolabi is with the department of Mathematics and Science from University Tecknologi, Malaysia and with the Federal Polytechnic KauraNamoda, Zamfara State, Nigeria.

Normah Maan works for the Mathematics Science Department at University Tecknologi, Malaysia.

Article submitted 2019-04-24. Resubmitted 2019-05-23. Final acceptance 2019-05-27. Final version published as submitted by the authors. 